encouraging families to consider a biopsy in asymptomatic patients. However, there are many occasions when serological tests have been omitted by clinicians, probably due to a lack of awareness.

Improvements are needed to reduce the length of time from diagnosis to dietician follow up, and follow up at 12 months needs to include repeat tTG assessment.

Finally, increased awareness is needed on the national policy to transition celiac patients to adult secondary care.

\section{G44(P) BIOELECTRIC IMPEDANCE VECTOR ANALYSIS (BIVA) AND CLINICAL OUTCOME IN HOSPITALISED CHILDREN}

\begin{abstract}
${ }^{1,2}{ }^{2}$ Roche, ${ }^{1} \mathrm{NE}$ Lara-Pompa, ${ }^{3} \mathrm{~S}$ Macdonald, ${ }^{4} \mathrm{~K}$ Fawbert, ${ }^{5} \mathrm{JE}$ Valente, ${ }^{4} \mathrm{~S}$ Hill, ${ }^{1} \mathrm{~J} C \mathrm{C}$ Wells, ${ }^{1} \mathrm{MS}$ Fewtrell. 'Childhood Nutrition Research Centre, UCL Great Ormond Street Institute of Child Health, London, UK; ${ }^{2}$ St Hughs College, University of Oxford, Oxford, UK; ${ }^{3}$ Dietetics Department, Great Ormond Street Hospital for Children NHS Trust, London, UK; ${ }^{4}$ Gastroenterology Department, Great Ormond Street Hospital for Children NHS Trust, London, UK; ${ }^{5}$ General Paediatrics Department, Great Ormond Street Hospital for Children NHS Trust, London, UK
\end{abstract}

\subsection{6/archdischild-2018-rcpch.42}

Aims Bioelectric impedance analysis (BIA) is a widely used, simple bedside technique, but clinical use is limited by the need to convert raw measurements to body composition, using equations that are potentially inappropriate. The use of the raw bioelectric impedance vectors (BIV), resistance (R), reactance $(\mathrm{Xc})$ and phase angle (PA) - suggested to indicate body fluid, cell mass and cell health respectively - may be an alternative for monitoring disease progression/treatment. However, clinical experience of BIV in children is limited and previous studies have not standardised for age. We investigated predictors of BIV and their ability to predict clinical outcomes in children with complex diagnoses admitted to a children's hospital.

Methods R, Xc and PA were measured using BODYSTAT Quadscan 4000 on admission in 70 children aged 4.6-16.8 years (mean 10.0). $\mathrm{R}$ and Xc were indexed by height $(\mathrm{H})$ and BIVSDS generated for age and sex using data from healthy children. Potential predictors (activity, wheelchair use, steroid treatment, enteral/parenteral nutrition); and clinical outcomes (greater-than-expected length-of-stay (LOS), complications (unplanned transfer to ICU, increased artificial nutrition, infection requiring antibiotics)) were recorded at discharge.

Results Mean R/HSDS was significantly higher (0.99 (SD 1.32)) and PASDS significantly lower $(-1.22(1.51))$ than the population mean, with a wide range for all BIVSDS. No significant predictors of BIVSDS were identified. BIVSDS were not significantly different in patients with or without adverse outcomes although R/HSDS was higher in children with increased LOS (mean difference mean difference 0.42 (95\% $\mathrm{CI}=-0.26$ to -1.11 ) or complications (mean difference 0.49 (95\% CI -0.34 to 1.33 ).

Conclusion This group of complex patients had abnormal mean BIVSDS suggestive of reduced hydration and poor cell health. However, factors considered as clinical predictors showed no significant association, and BIVSDS were not significantly related to clinical outcomes; possibly reflecting the necessary use of generic predictors and outcomes in this heterogeneous population. Children with adverse outcomes showed a trend towards higher R/HSDS, suggesting lower hydration. Further investigation in specific patient groups, including those with acute fluid shifts and using disease-specific outcomes, may help to better define the clinical role of BIV.

\section{G45(P) AN AUDIT OF WEANING PREMATURE INFANTS AT CORRECTED AGE AND ASSOCIATED ORAL FEEDING OUTCOMES}

S Russell, L Cairns, J Simpson, E Gentles. Neonatal Intensive Care Unit, Royal Hospital for Children, Glasgow, UK

10.1136/archdischild-2018-rcpch.43

Aim To assess feeding outcomes when weaning infants born $<32$ weeks gestation at 4-6 months corrected age (CGA).

Background There is limited evidence to support weaning preterm infants at 4-6 months CGA. Developmental Supportive Care is an integral part of treatment for premature infants at our site and we consider oral feeding to be a developmental skill. Our approach is to advise weaning at 4-6 months CGA, in line with other developmental skills. This is in contrast to the Consensus statement on weaning preterm infants produced in 2011.BLISS have recently(2017) updated their weaning guidelines and our audit provides evidence to support this. Our audit was carried out to ensure practice is associated with good oral feeding outcomes.

Method Data for 69 infants born $<32$ weeks gestation was collected using a specific proforma from August 2010 to October 2012, by a Speech and Language Therapist at developmental clinic.

Results 91\% (63 infants) had not started weaning by 4 months CGA (i.e. by 17 weeks CGA). Of the 9\% (6 infants) who had started weaning, median age was 14.5 weeks CGA (range: 13-16 weeks CGA). In the 63 infants weaned at CGA there were no reported problems in progression through textures up to 12 months CGA.

Conclusion In our cohort no increase in feeding related problems or aversions was identified in those weaned at 4-6 months CGA. We feel this supports our current weaning advice and highlights the importance of our Developmental Care programme. We acknowledge sample size is small and recognise that larger prospective data collection with a broader range of feeding related outcomes is required.

\section{G46(P) OUTCOME OF CHILDREN WITH INFLAMMATORY BOWEL DISEASE AFTER SURGERY}

N Kevlani. Paediatrics and Neonatology, Paras HMRI, Patna, India

\subsection{6/archdischild-2018-rcpch.44}

Aim To investigate the outcome after surgery in children with inflammatory bowel disease (IBD).

Method Case notes of patients who had surgery for IBD between November 1999 and January 2011 at a tertiary hospital in the UK were reviewed. Data related to relapses, acute readmissions, weights and heights one year before and up to a maximum of three years after surgery were collected. Mean Standard Deviation scores (SDS) were calculated for weights and heights. Outcomes were analysed using the paired test. Results 38 patients were eligible for the study. Of these case notes were available for 31 patients. 61\% $(n=19)$ had Crohn's Disease (CD) and 39\% $(n=12)$ had Ulcerative Colitis (UC). 
The commonest indication for surgery was failure of medical management (48\%). Surgical interventions included subtotal colectomy with ileostomy (18), extended right hemicolectomy (11), total colectomy (1) and limited ileal resection (1).

$74 \%(n=14)$ of CD patients had no relapses in the follow up period after surgery. The average number of relapses (in 12 patients for whom data was available) came down from 2.3 in the year before surgery to 0.4 in year after surgery with a mean reduction of $1.9 \quad(\mathrm{p}=0.0001 ; 95 \% \mathrm{CI}: 1.2$ to 2.7$)$. There was no statistically significant difference in the average number of acute readmissions between these periods. At one year after surgery the increase in mean SDS was 0.78 $(p=0.01$ CI: 0.2 to 1.3$)$ for weight and $0.3(p=0.002$; CI: 0.13 to 0.46 ) for height.

UC does not recur after total proctocolectomy. Hence there were no relapses after surgery. In the six patients for whom data was available, there was an insignificant increase in the average number of hospital admissions from 1 in the year before surgery to 1.5 in the year after (mean increase 0.5; $95 \% \mathrm{CI}: 1$ to $2 ; \mathrm{p}=0.45)$. At one year after surgery the change in mean SDS was $-0.1(p=0.5 ; C I$ : -0.4 to 0.2$)$ for weight and $0.18(\mathrm{p}=0.07$; CI: -0.02 to 0.4$)$ for height.

Conclusions There was improvement in growth and reduction in number of relapses after surgery in CD patients. For UC patients no significant improvement was seen either in terms of readmissions or growth

\section{G47(P) NON-CASEATING SPLENIC GRANULOMAS, AS THE FIRST MANIFESTATION OF VERY EARLY-ONSET INFLAMMATORY BOWEL DISEASE}

AP Rajendran, R Mardare, A Modi, M Ashworth, N Ramkumar, H Rollins. Paediatric Gastroenterology Department, Luton and Dunstable University Hospital, Luton, UK

10.1136/archdischild-2018-rcpch.45

Background Splenic granulomas (SG), are rare and a challenge in terms of diagnosis and treatment. There is no reported incidence for SG and they usually appear in young adults. Our patient presented with SG, as an extra-intestinal manifestation of Very Early-Onset Inflammatory Bowel Disease (VEO$I B D)$.

Case report We report the case of a 3 year old girl who developed multiple SG at the onset of Crohn's Disease (CD), making her, to our knowledge, the youngest patient reported to have this extra-intestinal manifestation of IBD.

She presented with a history of recurrent episodes of fever, vomiting, abdominal pain, weight loss and mouth ulcers. No infectious aetiology was found and she had normal immunological workup. Ultrasound (USS) of the abdomen showed hypoechoic splenic lesions. Splenic biopsy was performed, as despite an 8 week course of antibiotics there was no resolution of her symptoms and USS findings. Splenic biopsy showed non-caseating granulomas.

Her faecal calprotectin (FC) was raised, hence she had upper and lower gastrointestinal tract endoscopies and Video capsule endoscopy (VCE), which showed multiple ulcers throughout the small bowel, confirming a diagnosis of VEO-CD.

Results She was started on Modulen and Azathioprine, which improved her gut symptoms and the SG resolved on the USS. After the initial improvement her gut symptoms returned, so Infliximab was added to her management.
Four months after commencing Infliximab, she started developing symptoms again and her repeat FC was elevated. Repeat endoscopies and MRI reconfirmed the diagnosis of active CD. Both Azathioprine and Infliximab doses were increased, with some improvement of her symptoms. But Infliximab was later changed to Adalimumab as the Infliximab levels were persistently low. With just one loading dose of Adalimumab, she developing beningn intracranial hypertension, hence was discontinued. She is currently on Thalidomide and Azathioprine, thriving well, while being monitored with regular abdominal ultrasound.

Conclusion Patients with VEO-IBD usually do not respond to first line treatment, therefore biologics are often used early on in this disorder. Further studies on VEO-IBD need to be undertaken to help us manage this unusual disease entity.

\section{G48(P) INCIDENTAL FINDINGS IN MRI-ENTEROGRAPHY IN PAEDIATRIC PATIENTS DIAGNOSED WITH INFLAMMATORY BOWEL DISEASE}

N Basheer, SH Kanumakala, A Butt, V Lavanya. Paediatrics, Royal Alexandra Children Hospital, Brighton, UK

\subsection{6/archdischild-2018-rcpch.46}

Aims Although coincidental findings in inflammatory bowel disease (IBD) patients detected on MRI-enterography (MRE) have been extensively discussed in the adult population, only very few papers have discussed such findings in children. The primary aim of this study was to determine the frequency of incidental findings detected in children with confirmed IBD undergoing MRE for diagnostic/staging purposes. The secondary aim of the study was to evaluate the clinical impact of such incidental findings.

Methods All MRE performed at a single centre from January 2014 to December 2016 were retrospectively analysed; only patients with a confirmed diagnosis of IBD were included. A random selection of MRE without incidental findings was reviewed by a consultant radiologist to inform that incidental findings were not missed previously (double reporting). The medical notes of children with incidental findings were retrospectively reviewed to inform the clinical impact of such findings.

Results A total of 190 patients underwent MRE over the three years, but 102 patients with a confirmed diagnosis of IBD were only included. Incidental findings were noted in 16 patients (15.6\%); two were intestinal findings (probable small bowel intussusception) and the rest were extra intestinal findings. $50 \%$ ( 8 patients) of the incidental findings related to renal pathology (solitary kidney, cyst, duplex kidney, dilated pelvicalyceal system), $18.7 \%$ (3 patients) to spleen pathology (splenomegaly,cyst), $12.5 \%$ (2 patients) to prominent mesenteric lymphadenopathy, and $6.2 \%$ (1 patient) to gallbladder pathology (gallbladder stones) respectively.

In 7 patients $(43.7 \%$ ) (1 with kidney cyst, 2 with duplex kidney, 2 with dilated pelvicalyceal system, 2 with splenomegaly), further imaging studies (ultrasounds) were done. Incidental findings were re-confirmed in 4 of them; one patient with prominent splenomegaly required further haematology investigations and the other 3 patients with renal pathologies (1 with duplex kidney, 2 with dilated pelvicalyceal system) did not require further investigations. 